\title{
70 Jahre Kartographische Nachrichten - Ein Aufruf
}

\author{
Dennis Edler ${ }^{1}$
}

Published online: 8 October 2020

(c) The Author(s) 2020

Die Leserinnen und Leser dürften an dieser Stelle den Hauptschriftleiter, Mark Vetter, erwartet haben: Diesem gilt in diesem Sommer insbesondere eine Gratulation zu seinem Nachwuchs. Die nun anstehende Elternzeit sei ihm herzlich gegönnt!

In Vertretung für unseren Editor-in-Chief möchte ich die Gelegenheit nutzen, um mit diesem Editorial einen Aufruf an die KN-Leserschaft und damit an die potenziellen Autorinnen und Autoren von KN-Beiträgen zu starten: Die Kartographischen Nachrichten sind seit 1951 das wohl wichtigste Sprachrohr der wissenschaftlichen Kartographie in Deutschland, in Österreich und der Schweiz. Wer die fast 70 vollständigen Jahrgänge der Kartographischen Nachrichten in seinen Bücherregalen stehen hat oder in so manche Fachbibliothek blickt, sieht eine nahezu vollständige eindrucksvolle Dokumentation der Fachentwicklung der Kartographie im deutschsprachigen Raum, von der Nachkriegszeit bis ins 21. Jahrhundert. Dies verdeutlicht: Die Kartographischen Nachrichten haben „Impact“!

Der Begriff „Impact“" steht in der wissenschaftlichen Community jedoch heute nicht nur für fachliche Qualität über Jahrzehnte, sondern der viel zitierte und verwendete „Journal Impact Factor“ (JIF) ist ein international anerkanntes Maß, das den Einfluss und die Sichtbarkeit von Fachzeitschriften quantitativ bewertet und einen bibliometrischen Vergleich zwischen den Fachzeitschriften ermöglicht. In einer global vernetzten Wissenschaft wurden damit Wettbewerbsstrukturen etabliert, denen sich alle Zeitschriften - und sei die Tradition und fachliche Qualität zurückliegender Jahrzehnte noch so hoch - stellen müssen, um sich zukunftsfähig aufzustellen. Damit konkurrieren die $\mathrm{KN}$ heute u.a. mit neuen Zeitschriften, einschl. neuen „Mega-Journals“, die gänzlich auf ein Printverfahren verzichten und die Bedarfe der heutigen Wissenschaft (z.B. zeitsparendes Gutachterverfahren, schnelle OnlineVeröffentlichung nach Begutachtung, weltweite Sichtbarkeit veröffentlichter Beiträge über das Internet und eben auch: Qualitätssicherung durch einen ,Journal Impact Factor“) decken. Wenn es drittmittelwirksame Forschungsanträge - aus nachvollziehbaren Gründen - erfordern, aus jüngster Zeit gut sichtbare Ergebnisse aus der eigenen Arbeitsgruppe mit anzugeben, steigt unter Wissenschaftlerinnen und Wissenschaftlern die Bereitschaft, sich von klassischen Zeitschriften abzuwenden und sich neueren bedarfsgerechteren Strukturen und Publikationsmöglichkeiten (s.o.) anzuschließen. Ähnliche Gedanken mögen gerade auch jüngere Wissenschaftlerinnen und Wissenschaftler bei der Auswahl von Fachzeitschriften beeinflussen, wenn Promotions- und Habilitationsordnungen bei publikationsbasierten bzw. kumulativen Abschlussvorhaben in den Naturund Ingenieurswissenschaften Artikel in „Impact Journals“ vorsehen.

Selbstverständlich lässt sich die Sinnhaftigkeit dieser „Durchökonomisierung“ der Wissenschaftswelt hinterfragen und diskutieren, was auch häufig getan wird. Bei einigen neueren internationalen Impact-Journals garantieren bspw. Gutachterverfahren von weniger als sieben Tagen nicht unbedingt eine hinreichend fachliche Tiefe der eingeholten Gutachten, worunter die Qualität im Vergleich zu etablierten Strukturen leiden kann.

Beim Blick in die letzten KN-Jahrgänge wird deutlich, dass aus Deutschland, Österreich und der Schweiz weniger Arbeitsgruppen bzw. Autorinnen und Autoren, als es noch vor einem Jahrzehnt der Fall war, die Kartographischen

Dennis Edler

Dennis.Edler@ruhr-uni-bochum.de

1 Institute of Geography, Ruhr-University Bochum, Bochum, Germany 
Nachrichten mit Fachartikeln ausstatten. Um unsere gemeinsame Fachzeitschrift noch zukunftsfähiger aufzustellen, möchte ich an die Community appellieren: Publizieren Sie in dieser Zeitschrift und nutzen Sie den in den KN reichlich vorhandenen Wissensbestand als Quelle und Referenz. Damit können wir den „Impact“ der rund 70-jährigen Vergangenheit an die modernen Bedarfe anpassen und die $\mathrm{KN}$ zu einem international sichtbareren Journal mit ,Journal Impact Factor" machen! Dank Mark Vetter wurden mit dem Verlagswechsel zu Springer und den Möglichkeiten, wie Online-Vorabveröffentlichungen angenommener Artikel (Online First) und freien Abruf über die neue, moderne KN-Webseite (Open Access), ab 2019 Strukturen geschaffen, um sich dem Wettbewerb gut stellen zu können. In der Zitationsdatenbank ,Scopus“ sind die KN - dank vorausgehenden Bemühungen von Rolf Harbeck und Frank Dickmann - bereits vertreten.

Im Namen der Schriftleitung danke ich allen Autorinnen und Autoren der fachwissenschaftlichen Beiträge und des umfangreichen Vereinsteils für die Einreichungen! Die Redaktion freut sich auf Ihre Beiträge, um die Kartographischen Nachrichten weiterhin aktuell und attraktiv aufzustellen.

Herzliche Grüße und „Glückauf“ aus dem Ruhrgebiet!

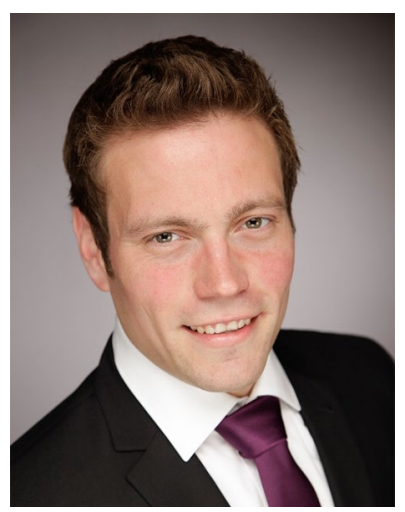

Dr. Dennis Edler
Open Access This article is licensed under a Creative Commons Attribution 4.0 International License, which permits use, sharing, adaptation, distribution and reproduction in any medium or format, as long as you give appropriate credit to the original author(s) and the source, provide a link to the Creative Commons licence, and indicate if changes were made. The images or other third party material in this article are included in the article's Creative Commons licence, unless indicated otherwise in a credit line to the material. If material is not included in the article's Creative Commons licence and your intended use is not permitted by statutory regulation or exceeds the permitted use, you will need to obtain permission directly from the copyright holder. To view a copy of this licence, visit http://creativecommons.org/licenses/by/4.0/. 